\title{
Fruits and vegetables are central to the richness of agriculture
}

"From herbs to apples, from walnuts to grapes, specialty crops are central to the richness of American agriculture." Such was the statement by the US Deputy Secretary of Agriculture, Kathleen Merrigan, when launching the Specialty Crop Research Initiative in October 2011. Through this initiative, the National Institute of Food and Agriculture (NIFA) will allocate 29 grants across 19 states, totaling \$46 million, to develop and share science-based tools to address the needs of America's specialty crop industry; including fruits and vegetables, tree nuts, dried fruits, and horticulture and nursery crops, including floriculture. Recognizing that "over the last 60 years, agriculture, including horticulture, has become increasingly reliant on science and technology to maintain profitable production", Kathleen Merrigan added that "these projects will help provide specialty crop producers with the information and tools they need to successfully grow, process and market safe and bigh quality products, supporting jobs and opportunities for Americans working in specialty crops".

Such an official position from a government is worth noting as an example of what is needed in many countries, especially in developing countries, where, too often, priority is given to major food such as cereals or meat. The role of the governments, through such engagements, should be one of the key components of the virtuous circle linking the production of fruits and vegetable to nutrition and better health. It should seek to stimulate private initiatives in production, packaging, processing and marketing of fruit and vegetables as part of public-private partnerships that are key to success. It is hoped that many more countries adopt such a position and consider fruits and vegetables as central to the richness of their agriculture.

Dr. Jacky Ganry Scientific Director of Fruits 


\section{Les fruits et légumes sont essentiels à la richesse de l'agriculture}

"Des herbes aux pommes, des noix aux raisins, les cultures spéciales sont essentielles à la richesse de l'agriculture américaine. "Telle est la déclaration de la Vice-secrétaire de l'Agriculture des États-Unis, Merrigan Kathleen, lors du lancement de l'Initiative de recherche sur les cultures spéciales en octobre 2011. Grâce à cette initiative, l'Institut national pour l'Alimentation et l'Agriculture (NIFA) va attribuer 29 subventions dans 19 états, totalisant 46 millions de dollars, pour développer et partager des outils scientifiques permettant de répondre aux besoins des États-Unis dans le secteur des cultures spéciales ; celles-ci regroupent les fruits et légumes, les noix, les fruits secs, et les cultures horticoles et plantes en pots, y compris la floriculture. Reconnaissant que "au cours des 60 dernières années, l'agriculture, y compris l'borticulture, est devenue de plus en plus tributaire de la science et de la technologie pour maintenir la production rentable ", Kathleen Merrigan a ajouté que "ces projets contribueront à fournir aux producteurs de cultures spéciales les informations et les outils dont ils ont besoin pour produire, transformer et commercialiser des produits sûrs et de qualité, en soutenant l'emploi et créant des oppor- tunités pour les Américains qui travaillent dans les cultures spéciales".

Une telle position officielle d'un gouvernement mérite d'être relevée comme un exemple de ce qui serait nécessaire dans de nombreux pays, et particulièrement dans les pays en développement, où, trop souvent, la priorité est donnée aux produits alimentaires majeurs comme les céréales ou la viande. Le rôle des gouvernements, à travers un tel engagement, devrait constituer l'un des éléments clés du cercle vertueux liant la production des fruits et légumes à la nutrition et à une meilleure santé. Il devrait viser à stimuler les initiatives privées dans la production, le conditionnement, la transformation et la commercialisation des fruits et légumes dans le cadre de partenariats publicprivé qui sont des éléments clés de la réussite. Il est à espérer que de nombreux autres pays adoptent une telle position et considèrent les fruits et légumes comme des éléments essentiels de la richesse de leur agriculture.

Dr. Jacky Ganry Directeur scientifique de Fruits 